\title{
Lifetimes and shape-coexisting states of ${ }^{99} \mathrm{Zr}$
}

P. Spagnoletti, ${ }^{1}$ G. Simpson, ${ }^{2}$ S. Kisyov, ${ }^{3}$ D. Bucurescu, ${ }^{3}$ J.-M. Régis, ${ }^{4}$ N. Saed-Samii, ${ }^{4}$ A. Blanc, ${ }^{5}$ M. Jentschel, ${ }^{5}$ U. Köster, ${ }^{5}$ P. Mutti, ${ }^{5}$ T. Soldner, ${ }^{5}$ G. de France, ${ }^{6}$ C. A. Ur, ${ }^{7}$ W. Urban,${ }^{8}$ A. M. Bruce,,${ }^{9}$ C. Bernards,${ }^{10}$ F. Drouet, ${ }^{2}$ L. M. Fraile, ${ }^{11}$ L. P. Gaffney, ${ }^{12}$ D. G. Ghită, ${ }^{3}$ S. Ilieva, ${ }^{13}$ J. Jolie, ${ }^{4}$ W. Korten,,${ }^{14}$ T. Kröll, ${ }^{13}$ S. Lalkovski, ${ }^{15}$ C. Larijarni, ${ }^{16,17}$ R. Lică, ${ }^{3}$ H. Mach, ${ }^{11,18}$ N. Mărginean, ${ }^{3}$ V. Paziy, ${ }^{11}$ Zs. Podolyák, ${ }^{16}$ P. H. Regan, ${ }^{16,17}$ M. Scheck, ${ }^{1}$ J. F. Smith, ${ }^{1}$ G. Thiamova, ${ }^{2}$ C. Townsley, ${ }^{16}$ A. Vancraeyenest, ${ }^{2}$ V. Vedia, ${ }^{11}$ N. Warr, ${ }^{4}$ V. Werner, ${ }^{10,13}$ and M. Zielińska ${ }^{14}$

${ }^{1}$ School of Engineering, University of the West of Scotland, Paisley PA1 2BE, United Kingdom

${ }^{2}$ LPSC, Université Grenoble Alpes, CNRS/IN2P3, Institut National Polytechnique de Grenoble, 38026 Grenoble Cedex, France

${ }^{3}$ Horia Hulubei National Institute of Physics and Nuclear Engineering (IFIN-HH), 077125 Bucharest, Romania

${ }^{4}$ Institut für Kernphysik der Universität zu Köln, Zülpicher Straße 77, 50937 Köln, Germany

${ }^{5}$ Institut Laue-Langevin, 71 avenue des Martyrs, 38042 Grenoble Cedex 9, France

${ }^{6}$ Grand Accélérateur National d'Ions Lourds, CEA/DRF-CNRS/IN2P3, Boulevard Henri Becquerel, 14076 Caen, France

${ }^{7}$ INFN, via Marzolo 8, 35131 Padova, Italy

${ }^{8}$ Faculty of Physics University of Warsaw, ulica Pasteura 5, 02-093 Warsaw, Poland

${ }^{9}$ SCEM, University of Brighton, Lewes Road, Brighton BN2 4GJ, United Kingdom

${ }^{10}$ WNSL, Yale University, P.O. Box 208120, New Haven, Connecticut 06520-8120, USA

${ }^{11}$ Grupo de Fisica Nuclear \& IPARCOS, Universidad Complutense de Madrid, CEI Moncloa, 28040 Madrid, SPAIN

${ }^{12}$ EP Department, ISOLDE, CERN, Geneva, Switzerland

${ }^{13}$ Institut für Kernphysik, TU Darmstadt, Schlossgartenstraße 9, 64289 Darmstadt, Germany

${ }^{14}$ CEA de Saclay, IRFU, 91191 Gif-sur-Yvette, France

${ }^{15}$ Faculty of Physics, University of Sofia, 1164 Sofia, Bulgaria

${ }^{16}$ Department of Physics, University of Surrey, Guildford GU2 7XH, United Kingdom

${ }^{17}$ National Physical Laboratory, Teddington, Middlesex TW11 OLW, United Kingdom

${ }^{18}$ National Centre for Nuclear Research, ulica Hoza 69, Warsaw, Poland

(Received 14 January 2019; revised manuscript received 18 April 2019; published 16 July 2019)

\begin{abstract}
Lifetimes of intermediate-spin states in two rotational bands of ${ }^{99} \mathrm{Zr}$ have been measured. These states were populated following the neutron-induced fission of ${ }^{235} \mathrm{U}$ at the PF1B beamline of the Institut Laue-Langevin, Grenoble, during the EXILL-FATIMA campaign. The nucleus ${ }^{99} \mathrm{Zr}_{59}$ exhibits shape coexistence and lies precisely on the border of an abrupt change in ground-state deformation when going from $N=58$ to $N=60$, making its study interesting for understanding the mechanisms involved in the rapid onset of deformation here. The $B(E 2)$ values extracted for decays in the $v 3 / 2$ [541] band allow quadrupole deformations of $\beta_{2}=0.34(1)$ and $0.26(3)$ to be determined for the 821.6- and 1236.6-keV members, whereas $\beta_{2}=0.32(3)$ was found for the $850.5-\mathrm{keV}$ member of the $v 3 / 2$ [411] band. Some of the excited states known in ${ }^{99} \mathrm{Zr}$ have been reasonably described with interacting boson-fermion model (IBFM) calculations. Type-II shell evolution is proposed to play a major role in modifying single-particle energies in ${ }^{99} \mathrm{Zr}$.
\end{abstract}

DOI: 10.1103/PhysRevC.100.014311

\section{INTRODUCTION}

Nuclei of the neutron-rich $A \approx 100$ region possess a wide variety of shape phenomena. A rapid change in ground-state deformation between the spherical ${ }^{96} \mathrm{Zr}$, weakly deformed ${ }^{98} \mathrm{Zr}$ [1], and strongly deformed ${ }^{100} \mathrm{Zr}$ [2] is well known. This discovery was surprising as the properties of the atomic nucleus generally evolve smoothly as a function of neutron

Published by the American Physical Society under the terms of the Creative Commons Attribution 4.0 International license. Further distribution of this work must maintain attribution to the author(s) and the published article's title, journal citation, and DOI. or proton number. The $Z=38\left(\pi p_{3 / 2}\right)$ and $40\left(\pi p_{1 / 2}\right)$ and the $N=50\left(v g_{9 / 2}\right), 56\left(v d_{5 / 2}\right)$, and $58\left(v s_{1 / 2}\right)$ spherical subshell closures, combined with a weak integrated residual proton-neutron interaction, mean that from $N=50$ to 58 the low energy structure of the $\mathrm{Sr}$ and $\mathrm{Zr}$ isotopes resembles that of a semimagic nucleus. At $N=60$ a strongly (prolate) deformed ground-state rotational band develops and forms the first few states. This shape change is more gradual across $N=58-60$ for the higher- $Z$ Mo-Pd nuclei of the region, which exhibit triaxiality [3-6]. At lower $Z$ values the $N \geqslant 60$ nuclei ${ }^{96,98,100} \mathrm{Kr}$ have recently been shown to possess considerably less collectivity, due to reduced $\pi g_{9 / 2}$ occupation and prolate-oblate shape coexistence $[7,8]$. The rapid nature of this ground-state deformation change has made this region a challenge to study theoretically. Furthermore, these neutron-rich 
nuclei are often far from stability and difficult to access experimentally.

A variety of theoretical explanations exist to explain this shape change including the crossing of two different meanfield configurations; one governed by spherical shell effects and the other a deformed intruder containing multiple particlehole excitations, dominated by the integrated residual protonneutron interaction $[9,10]$. Experimental evidence for coexisting spherical and deformed configurations exists for ${ }^{96-98} \mathrm{Sr}$ [11,12], ${ }^{96-104} \mathrm{Y}$ [13-15], and ${ }^{98-100} \mathrm{Zr}$ [10,16-19]. A strong interaction between occupied $\pi g_{9 / 2}-\nu g_{7 / 2}$ spin-orbit partners has been proposed to play a major role in obliterating the $N=$ 56,58 and $Z=38,40$ spherical subshell closures, leading to collective motion [20-22]. Occupation of high- $j$, low- $\Omega$ deformation driving $v h_{11 / 2}$ orbitals [21], along with the presence of deformed shell gaps at $Z=38, N=60$ [4,13], has also been shown to help stabilize deformation here. The rapid onset of the ground-state deformation is an example of quantum shape phase transition [23]. Recently type-II shell evolution [24] has been proposed to explain the onset of collectivity in this region [25]. In this mechanism specific particle-hole excitations for excited levels modify the underlying shellstructure due to the changed occupation probabilities altering the strength of the tensor force.

For the $\mathrm{Zr}$ isotopes the latter mechanism occurs once the $v d_{5 / 2}$ subshell approaches full occupation and neutrons can then first occupy the $v g_{7 / 2}$ and $v h_{11 / 2}$ orbits. Proton particlehole excitations provide initial $\pi g_{9 / 2}$ subshell occupation. These then interact strongly and attractively with neutrons situated in both the $v g_{7 / 2}$ spin-orbit partner and $v h_{11 / 2}$ orbits. The tensor and central forces act coherently on the $\nu g_{7 / 2}$ orbit, lowering its monopole energy, which in turn results in higher occupancy. Although the tensor and central forces act incoherently for the $\pi g_{9 / 2}-v h_{11 / 2}$ coupling, the net interaction is still attractive. The energy of the $\pi g_{9 / 2}-\pi p_{1 / 2}$ subshell gap is then reduced, further increasing its filling. Higher occupancy of the $\pi g_{9 / 2}$ orbit leads to the creation of more holes in the $\pi p f$ shell, further increasing collectivity. Evidence for this feedback mechanism presents itself beyond $N=56$, once the $v d_{5 / 2}$ subshell approaches full occupation and neutrons can first enter the $v g_{7 / 2}$ and $v h_{11 / 2}$ orbits. Quasirotational bands appear at 1581.6 and $854.0 \mathrm{keV}$ in ${ }^{96,98} \mathrm{Zr}$, which drop further in energy and increase in collectivity in ${ }^{100} \mathrm{Zr}$, to become the ground-state rotational band.

It is of particular interest to study the properties of shapecoexisting structures at the spherical-deformed border $(N=$ 59), which may allow additional insights into the underlying mechanisms driving the shape change. To date no coherent theoretical explanation exists describing all shape coexisting features found in this region, even though it contains some of the best known cases in the whole nuclear landscape [26]. In ${ }^{99} \mathrm{Zr}$ low-lying spherical states coexist with well-deformed rigid rotational bands situated at $0.6-1.0 \mathrm{MeV}[10,17,18,27]$. An absence of reliable and pertinent experimental data on the rotational bands of ${ }^{99} \mathrm{Zr}$ has so far hampered any theoretical interpretations. Different quadrupole moments have been reported for the $v 9 / 2[404]$ and $\nu 3 / 2$ [541] bands of ${ }^{99} \mathrm{Zr}$ $[17,18]$, implying that the rigidity of its core is sensitive to the occupation of specific orbitals. Furthermore, there is, to date, no evidence for oblate shapes expected to exist and play a significant role in the rapid onset of ground-state deformation here. For this reason we have chosen to study the shapes of the rotational bands in ${ }^{99} \mathrm{Zr}$.

Measurements of the electromagnetic transition rates between different states in the bands of odd- $A$ nuclei can give crucial information on collectivity, or help identify the unpaired orbital. The reduced $E 2$ transition rate, $B(E 2)$, is directly related to quadrupole collectivity. This quantity can be derived from the measured lifetime of a stretched intraband E2 transition. Alternatively, the underlying single-particle structure of a band can be probed by measurements of intraband $B(M 1)$ values, which are often orbital dependent. In the present study the lifetimes of excited states in ${ }^{99} \mathrm{Zr}$ have been measured. These nuclei were populated following the cold-neutron induced fission of ${ }^{235} \mathrm{U}$. Previously, the $\beta$ decay of mass-separated ${ }^{99} \mathrm{Y}$ ions was used to populate ${ }^{99} \mathrm{Zr}$ for lifetime measurements [10]. The $5 / 2^{+}$spin of the ground state of ${ }^{99} \mathrm{Y}$ [15], originating from the occupation of a $\pi 5 / 2$ [422] Nilsson orbital, permitted only limited access to members of the rotational bands of ${ }^{99} \mathrm{Zr}$. The prompt-fission reaction produces nuclei, on average, with intermediate-spin states $(6 \hbar-8 \hbar)$, allowing lifetime measurements of levels with higher spins than those fed following $\beta$ decay.

\section{EXPERIMENT}

Lifetimes of excited states in ${ }^{99} \mathrm{Zr}$ were measured via the fast-timing technique. This uses $\gamma(E, t)-\gamma(E, t)$ coincidences for direct timing measurements and relies on the good time resolution $(\approx 200 \mathrm{ps})$ of $\mathrm{LaBr}_{3}(\mathrm{Ce})$ detectors to access the lifetimes of states in the 10 ps-to-ns time range. Cold-neutron induced fission of ${ }^{235} \mathrm{U}$ produces secondary neutron-rich nuclei in the $A \approx 100$ region with average spins of $6 \hbar-8 \hbar$. It is therefore a suitable reaction to populate the excited states of interest in ${ }^{99} \mathrm{Zr}$, especially as this nucleus is situated at one peak of the fission-yield distribution. As some 100 fission fragments are reasonably well produced in this reaction, the modest energy resolution of the $\mathrm{LaBr}_{3}(\mathrm{Ce})$ detectors is not sufficient to uniquely select a $\gamma$-ray cascade of interest. This requires additional $\gamma$-ray detection using Ge detectors with their superior energy resolution.

The experiment was performed at the PF1B cold-neutron guide [28] of the high-flux reactor of the Institut LaueLangevin, Grenoble, within the framework of the EXILLFATIMA campaign [29]. The collimated neutron beam, with a flux $\Phi \approx 10^{8} \mathrm{n} /\left(\mathrm{cm}^{2} \mathrm{~s}\right)$, was $\approx 1 \mathrm{~cm}^{2}$ in area [30] and induced fission in a $0.8-\mathrm{mg}^{235} \mathrm{UO}_{2}\left(0.675 \mathrm{mg}{ }^{235} \mathrm{U}\right)$ target. The target was sandwiched between two $25-\mu \mathrm{m}$-thick Be backings, in order to stop the fission fragments in a time of just a few ps. This minimized the Doppler broadening of any emitted prompt $\gamma$ rays. The target was placed at the center of the EXILL-FATIMA $\gamma$-ray detector array, which consisted of 8 EXOGAM clover $\mathrm{Ge}$ and $16 \mathrm{LaBr}_{3}(\mathrm{Ce})$ detectors [29]. The data were acquired during a two-week measurement period and sorted offline into $\gamma(\mathrm{Ge})-\gamma\left(\mathrm{LaBr}_{3}\right)-\gamma\left(\mathrm{LaBr}_{3}\right)$ coincidence events, occurring within a 120 -ns time window. Timing signals of the $\mathrm{LaBr}_{3}(\mathrm{Ce})$ detectors were recorded in analog 


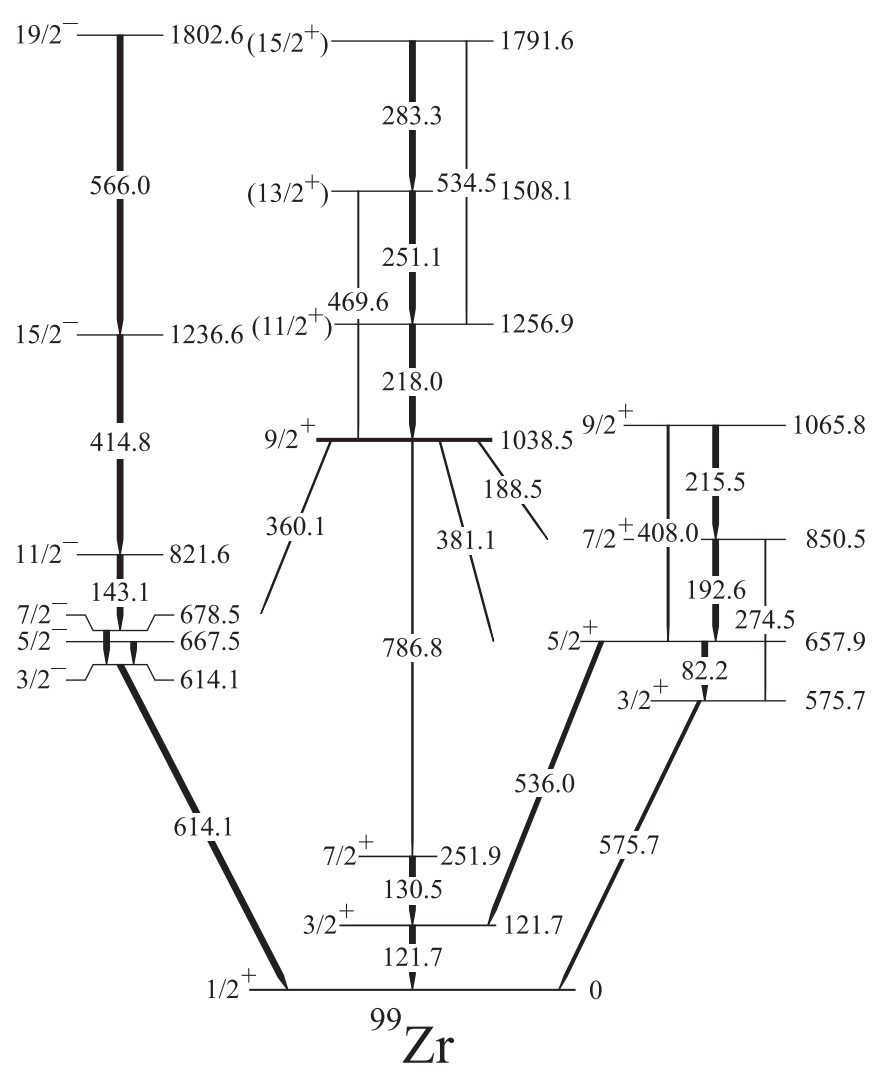

FIG. 1. Partial decay scheme of ${ }^{99} \mathrm{Zr}$. Transition and level energies are taken from Ref. [18]. Some known weak decay branches are not shown.

time-to-amplitude converters (TACs) with a $50 \mathrm{~ns}$ range. More details on the experiment can be found in Ref. [29].

\section{RESULTS}

A partial level scheme of ${ }^{99} \mathrm{Zr}$, containing the levels and decays of interest for the present work, is shown in Fig. 1. Here the level and transition energies are taken from Ref. [31].

The lifetime of the 121.7-keV level has been reported to be measured several times [31] and, therefore, constitutes a good test case. Furthermore several lifetimes measured from this data set have been reported previously [32-34]. The 192.6$536.0-121.7-\mathrm{keV}$ cascade was used for this. The spectra presented in Fig. 2 were obtained by gating on the 192.6$\mathrm{keV}$ transition (blue), using a background gate at $208 \mathrm{keV}$ (red), with the Ge detectors, and by gating on the $536.0-\mathrm{keV}$ transition with the $\mathrm{LaBr}_{3}(\mathrm{Ce})$ detectors. The corresponding time spectra (see Fig. 3) were produced with an additional gate using the $\mathrm{LaBr}(\mathrm{Ce})$ detectors on the $121-\mathrm{keV}$ transition and shows that the background is not entirely prompt and therefore contributes to the slow component of the time spectrum. The background is subtracted and the slope is measured. This was performed for both the delayed and antidelayed time spectra and a weighted average is given. The delayed time spectrum corresponds to a time-to-amplitude converter (TAC) being started with a transition which feeds the level of interest and stopped by a transition decaying from the level of interest. The

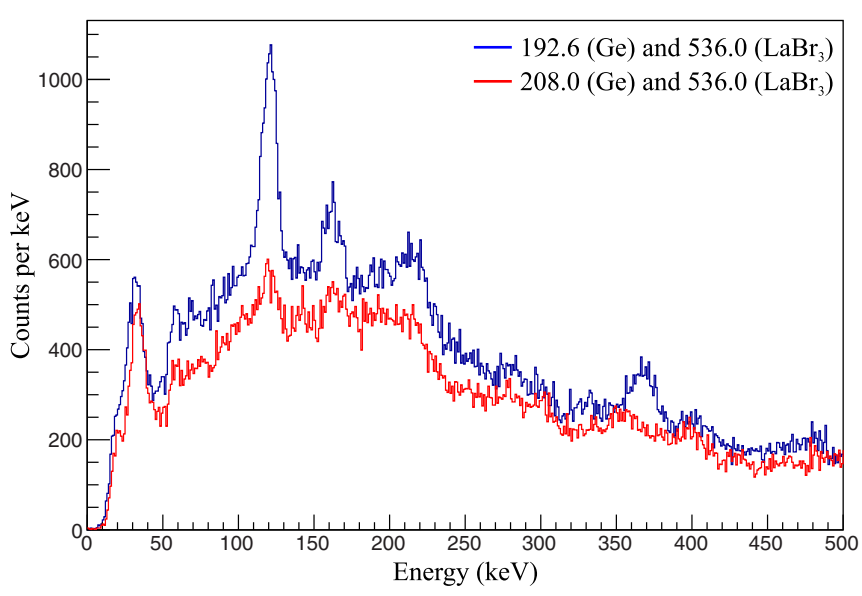

FIG. 2. Energy spectra obtained when gating on the 536.0-keV transition with the $\mathrm{LaBr}_{3}(\mathrm{Ce})$ detectors and the $192.6-\mathrm{keV}$ transition (blue) and a background energy of $208.0 \mathrm{keV}$ with the Ge detectors, respectively.

reverse is true for the antidelayed time spectrum. The value of $\tau=1550(90)$ ps obtained in the present work agrees well with the value of $\tau=1544(43)$ ps reported in the compilation of Ref. [31], while a value of $\tau=1880(120)$ ps is obtained

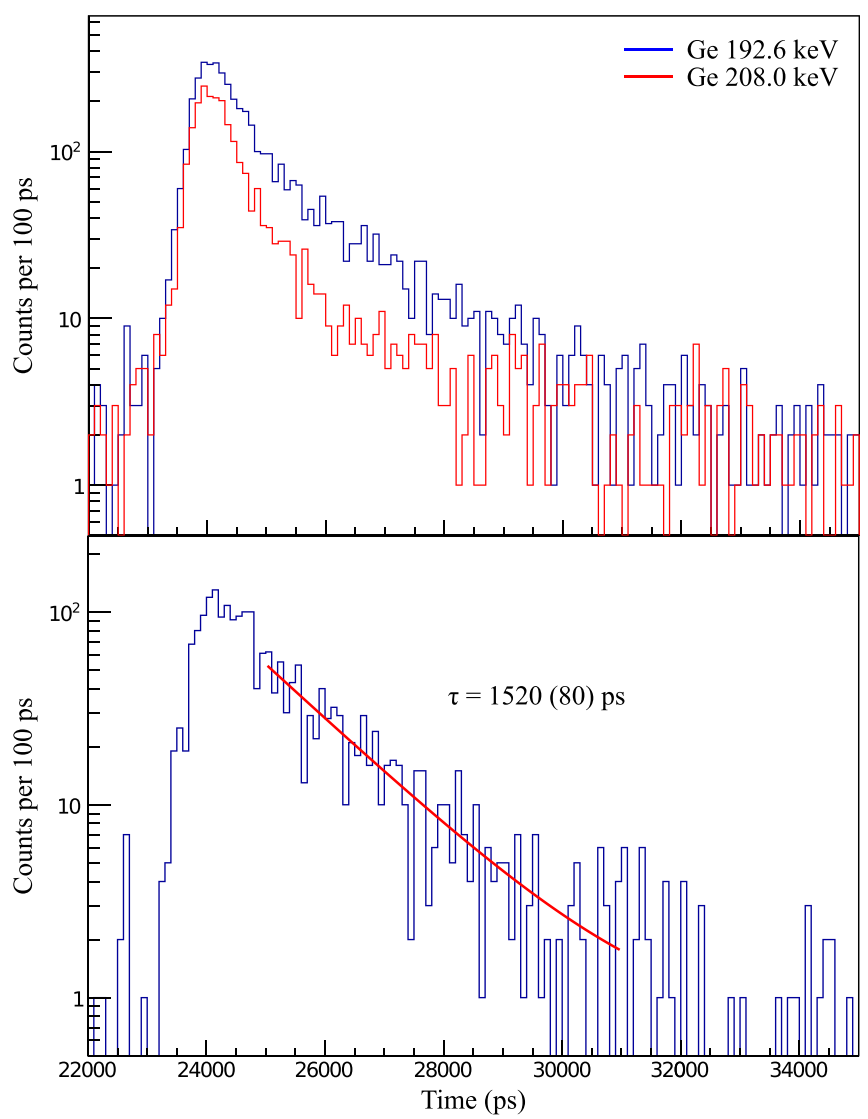

FIG. 3. Delayed time spectra obtained when gating on the 536.0and $121.7-\mathrm{keV}$ transitions with the $\mathrm{LaBr}_{3}(\mathrm{Ce})$ detectors and additional gates on the 192.6-keV transition (blue) and a background energy of $208.0 \mathrm{keV}$ (red). 
TABLE I. Gates set on Ge and $\mathrm{LaBr}_{3}(\mathrm{Ce})$ detectors for lifetime $(\tau)$ measurements and the results obtained. The subscripts $i j$ and $j i$ correspond to the gate orders start/stop and stop/start, respectively. Lifetimes above $1 \mathrm{~ns}$ were determined using the slope (SL) method and lifetimes below 1 ns were determined using the centroid difference $(\mathrm{CD})$ method.

\begin{tabular}{lccccc}
\hline \hline \multirow{2}{*}{$E_{i}(\mathrm{keV})$} & \multicolumn{2}{c}{$\gamma$-ray Gates $(\mathrm{keV})$} & \multirow{2}{*}{$\tau(\mathrm{ps})$} & \multirow{2}{*}{ Method } \\
\cline { 2 - 4 } & $\mathrm{Ge}$ & $\mathrm{LaBr}_{i j}$ & $\mathrm{LaBr}_{j i}$ & & \\
\hline 121.7 & 192.6 & 536.0 & 121.7 & $1550(90)$ & $\mathrm{SL}$ \\
657.9 & 121.7 & 192.6 & 536.0 & $35(13)$ & $\mathrm{CD}$ \\
821.6 & 566.0 & 415.0 & 143.0 & $3900(200)$ & $\mathrm{SL}$ \\
850.5 & $121.7,536.0$ & 215.5 & 192.6 & $100(25)$ & $\mathrm{CD}$ \\
1236.6 & 143.0 & 566.0 & 415.0 & $40(7)$ & $\mathrm{CD}$ \\
\hline \hline
\end{tabular}

without employing any background subtraction. This method was also employed to measure the lifetime of the $2_{1}^{+}$state in ${ }^{146} \mathrm{Ba}$. A value of $\tau=1260(40) \mathrm{ps}$ is obtained, which agrees well with the value of $\tau=1240$ (40) ps reported in the compilation of Ref. [35]

Measurements of the lifetimes of four other states of ${ }^{99} \mathrm{Zr}$ were possible. The various gates used are listed in Table I, along with the lifetimes obtained. For the $850.5-\mathrm{keV}$ state a quadruple $\mathrm{Ge}-\mathrm{Ge}-\mathrm{LaBr}_{3}-\mathrm{LaBr}_{3}$ coincidence was required to achieve the necessary selectivity. A summary of the results obtained is presented in Table I. With the exception of the value for the $121.7-\mathrm{keV}$ level, all other lifetimes are reported for the first time.

Spectra obtained when gating on the 121.7- and 192.6-keV transitions in the $\mathrm{Ge}$ and $\mathrm{LaBr}_{3}(\mathrm{Ce})$ detectors are shown in Fig. 4. Here one can clearly see the $536.0-\mathrm{keV}$ transition depopulating 657.9-keV level in both the $\mathrm{Ge}$ and $\mathrm{LaBr}_{3}(\mathrm{Ce})$ detectors.

The lifetime of the $657.9-\mathrm{keV}$ level was measured using the centroid difference technique. The lifetime is determined by the centroid difference in the delayed and antidelayed time spectra, which are shown in Fig. 5. When there is no background contribution the lifetime is related to the centroid

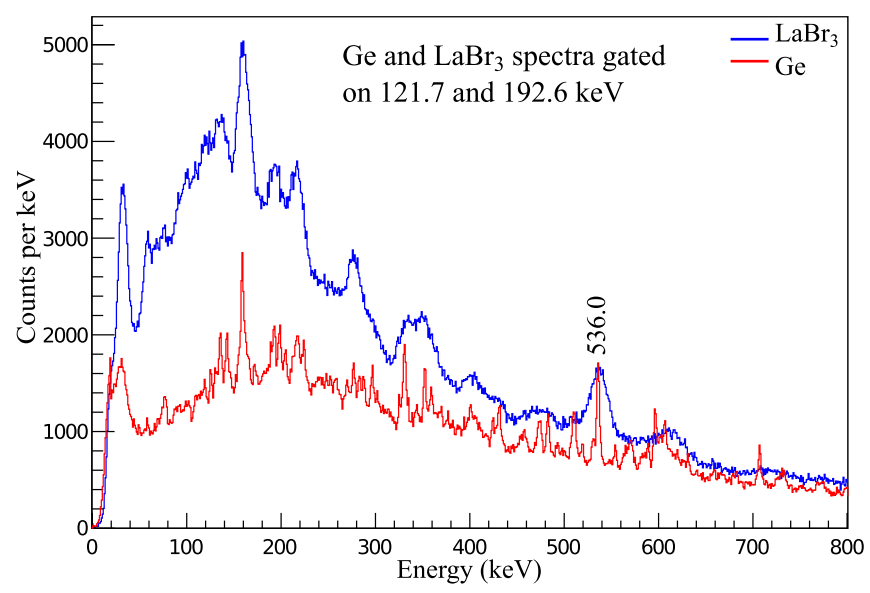

FIG. 4. Energy spectra obtained when gating on the 121.7- and 192.6-keV $\gamma$ rays in the $\mathrm{Ge}$ and $\mathrm{LaBr}_{3}(\mathrm{Ce})$ detectors, respectively.

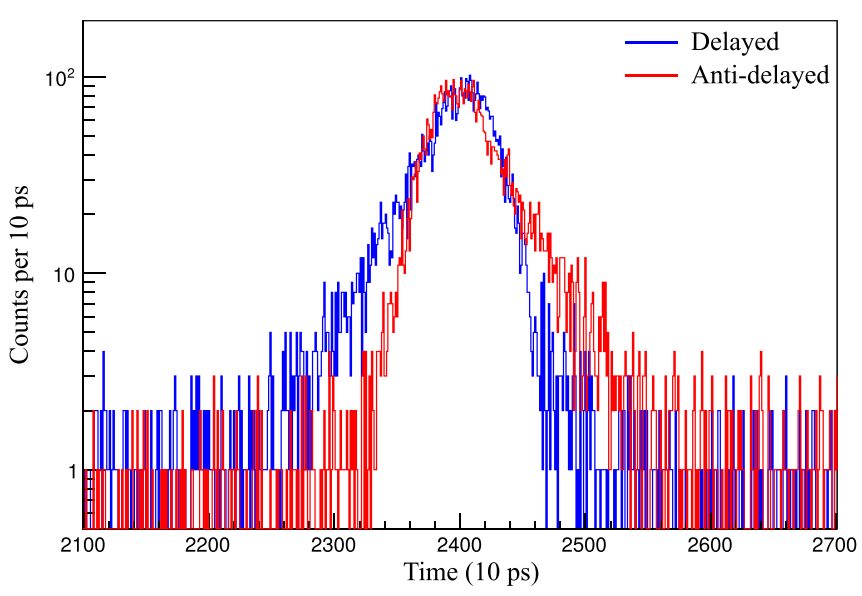

FIG. 5. Time spectra of the 657.9-keV level obtained with $\gamma$-ray gates on the 121.7-keV transition (Ge) and on the 536.0- and 192.6$\mathrm{keV} \gamma$ rays $\left(\mathrm{LaBr}_{3}\right)$.

difference by the equation

$$
\Delta C_{\mathrm{FEP}}=\mathrm{PRD}+2 \tau,
$$

where FEP stands for full-energy peak and PRD is the prompt response difference. The PRD describes the combined $\gamma-\gamma$ time walk of the setup [36].

In the case where the background contribution to the time spectra are not negligible, the centroid difference between the time distributions corresponding to the full-energy peaks, $C_{\mathrm{FEP}}$, is related the the experimental centroid difference, $C_{\text {exp }}$, by the relation

$$
\left.\left.\Delta C_{\mathrm{FEP}}=\Delta C_{\mathrm{exp}}+\frac{1}{2}\left[t_{\text {corr. }} \text { (feeder }\right)+t_{\text {corr. }} \text { (decay }\right)\right],
$$

where

$$
t_{\text {corr. }} \text { (feeder) }=\left[\frac{\Delta C_{\text {exp }}-\Delta C_{b g}}{P / B}\right]_{\text {feeder }},
$$

and

$$
t_{\text {corr. }} \text { (decay) }=\left[\frac{\Delta C_{\text {exp }}-\Delta C_{b g}}{P / B}\right]_{\text {decay }} .
$$

The term $\Delta C_{b g}$ is the centroid difference of the background and is determined analytically for both transitions. The analytical corrections for both the feeding and decaying transitions are shown in Figs. 6 and 7, respectively. The term $P / B$ is the peak-to-background ratio. A thorough description of this method is presented in Refs. [33,34]

Transition branching ratios from [31] and corrections for internal conversion [37] were used to convert the measured level lifetimes into partial stretched $E 2$ lifetimes $\left(\tau_{E 2}\right)$. Reduced transition rates were then calculated using the formula

$$
B(E 2)=\frac{450 \hbar}{24 \pi e^{2} b^{2} \tau_{E 2}}\left(\frac{\hbar c}{E_{\gamma}}\right)^{5} .
$$

From the $B(E 2)$ values transition quadrupole moments, $Q_{t}$, can be derived in the rigid-rotor framework using the formula

$$
Q_{t}^{2}=\frac{16 \pi}{5} \frac{B(E 2)}{\left\langle J_{i} K 20 \mid J_{f} K\right\rangle^{2}},
$$




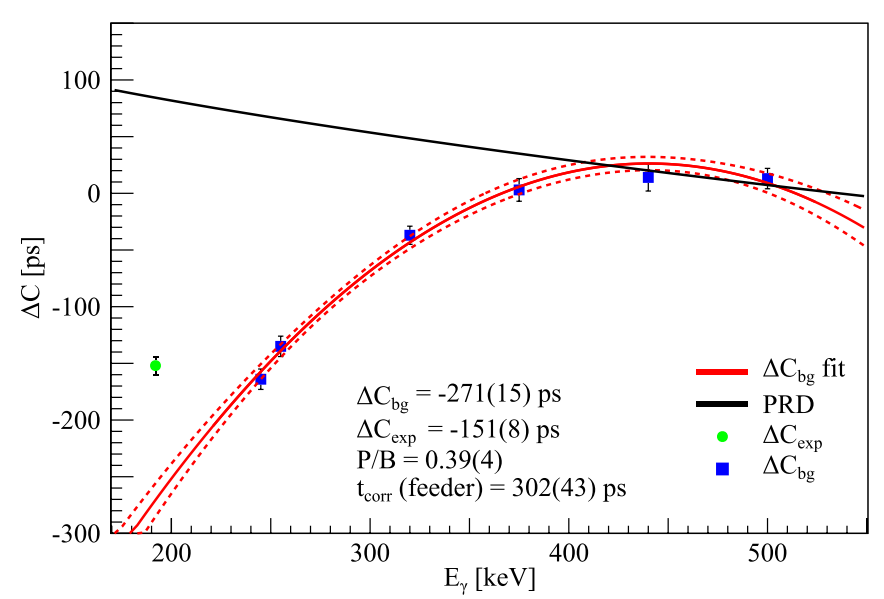

FIG. 6. Centroid distribution as a function of $\mathrm{LaBr}_{3}$ energy with gates set on the $121.7-\mathrm{keV}$ transition $(\mathrm{Ge})$ and on the $536.0-\mathrm{keV} \gamma$ ray $\left(\mathrm{LaBr}_{3}\right)$.

where $J_{i}$ is the initial spin, $J_{f}$ is the final spin, and $K$ is the projection of the aligned angular momentum on the symmetry axis. Deformation parameters, $\beta_{2}$, can be determined using

$$
\beta_{2}=\frac{91.7 Q_{0}}{Z A^{2 / 3}}
$$

with $Q_{t}=Q_{0}$.

For $\Delta J=1$ transitions the experimental partial $M 1$ lifetime $\left(\tau_{M 1}\right)$ is related to the reduced transition rate by

$$
B(M 1)=\frac{9 \hbar}{16 \pi \mu_{N}^{2} \tau_{M 1}}\left(\frac{\hbar c}{E_{\gamma}}\right)^{3}
$$

In the case of the $\left(3 / 2^{+}\right)$band built on either the $575.7-$ [18] or $657.9-\mathrm{keV}$ [27] states, conflicting spin assignments exist. The present work adopts the $J^{\pi}$ assignments from Ref. [18] and provides experimental evidence to support this choice in Sec. IV. A rigid-rotor analysis of the $\frac{\Delta J=2}{\Delta J=1}$

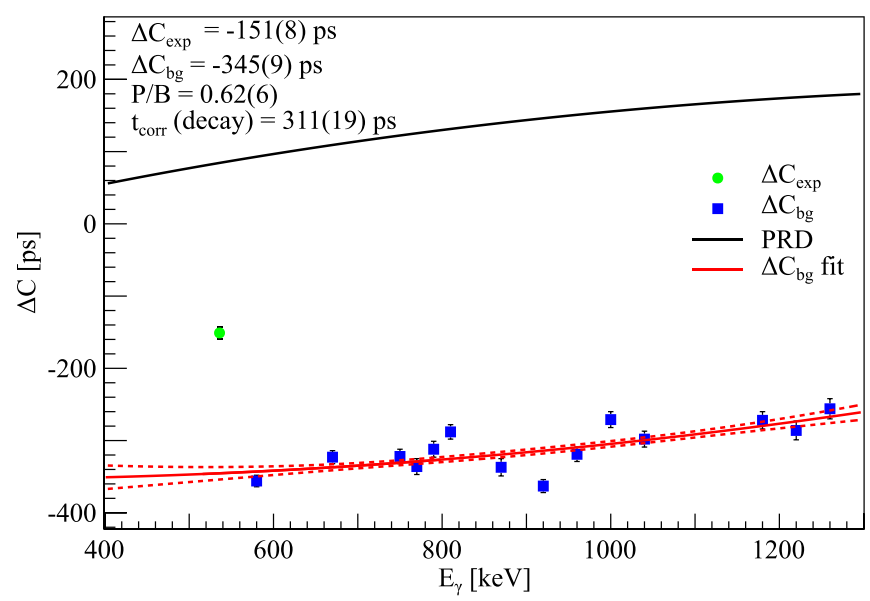

FIG. 7. Centroid distribution as a function of $\mathrm{LaBr}_{3}$ energy with gates set on the $121.7-\mathrm{keV}$ transition $(\mathrm{Ge})$ and on the $192.6-\mathrm{keV} \gamma$ ray $\left(\mathrm{LaBr}_{3}\right)$.
TABLE II. Level energies $E_{i}$, transition energies $E_{\gamma}$, level lifetimes, and reduced transition rates $B(\sigma L)$ determined for states of ${ }^{99} \mathrm{Zr}$ in the present work. All $\Delta J=0, \pm 1$ transitions are assumed to be pure $M 1$.

\begin{tabular}{lrccc}
\hline \hline$E_{i}(\mathrm{keV})$ & $E_{\gamma}(\mathrm{keV})$ & $J_{i} \rightarrow J_{f}$ & $\sigma L$ & $B(\sigma L)($ W.u. $)$ \\
\hline 121.7 & 121.7 & $3 / 2^{+} \rightarrow 1 / 2^{+}$ & $M 1$ & $0.0101(7)$ \\
657.9 & 82.2 & $5 / 2^{+} \rightarrow 3 / 2^{+}$ & $M 1$ & $0.042(21)$ \\
& 536.0 & $5 / 2^{+} \rightarrow 3 / 2^{+}$ & $M 1$ & $0.0047(20)$ \\
821.6 & 143.0 & $11 / 2^{-} \rightarrow 7 / 2^{-}$ & $E 2$ & $99(6)$ \\
850.5 & 192.6 & $7 / 2^{+} \rightarrow 5 / 2^{+}$ & $M 1$ & $0.032(10)$ \\
& 274.5 & $7 / 2^{+} \rightarrow 3 / 2^{+}$ & $E 2$ & $46(12)$ \\
1236.6 & 415.0 & $15 / 2^{-} \rightarrow 11 / 2^{-}$ & $E 2$ & $60(11)$ \\
\hline \hline
\end{tabular}

branching ratios allows the mixing ratio $\delta$ to be estimated for intraband transitions. For either scenario the $E 2$ contribution to a $\Delta J=1$ transition was calculated to be at most $23-\%$ and more typically $\approx 5 \%$. These estimations agree with the experimentally measured $\delta$ values for transitions in the $v 3 / 2$ [411] band of the neighboring ${ }^{101} \mathrm{Zr}[38,39]$. Therefore $\Delta J=1$ transitions in the $\left(3 / 2^{+}\right)$band were considered to be pure $M 1$ in nature in the analysis. Values of $B(M 1)=0.032(10)$ and $B(M 1)=0.042(21)$ W.u. were found for the 192.6- and $82.2-\mathrm{keV}$ transitions, respectively, in the $\gamma$ cascade of the 850.5-657.9-575.7-keV levels. A summary of the reduced transition probabilities measured in this work is given in Table II. Calculations of deformation parameters for members of the $v 3 / 2[541]$ and $v 3 / 2$ [411] bands are presented in Table III.

\section{DISCUSSION}

\section{A. 575.7-657.9-850.5-keV levels}

The exact nature of the 575.7- and 657.9-keV levels is uncertain. A positive parity was assigned to these states based on measured $\log f t$ values from the $\beta$ decay of ${ }^{99} \mathrm{Y}[10,40]$. In [17] these two levels were presented as the bandheads of two separate $K=3 / 2^{+}$and $5 / 2^{+}$decoupled bands. This was then revised to them being the $\left(3 / 2^{+}\right)$and $\left(5 / 2^{+}\right)$members of the same $K=3 / 2^{+}$band [18]. This interpretation is supported by an observed transition between the $K=9 / 2^{+}$isomer at $1038.5 \mathrm{keV}$ and the $657.9-\mathrm{keV}$ level with energy $381 \mathrm{keV}$. However, Lhersonneau et al. later noted that the $82.2-\mathrm{keV}$ $K+1 \rightarrow K$ transition connecting them appeared to be too weak to be an intraband transition [40]. In a separate work the $657.9-\mathrm{keV}$ state was proposed to be a $3 / 2^{+}$bandhead, with mixed $v 3 / 2[411]$ and $v 3 / 2[422]$ parentage [27], though the

TABLE III. Level energies $E_{i}$, transition energies $E_{\gamma}$, reduced transition rates $B(E 2)$, and deformation parameters determined for states of ${ }^{99} \mathrm{Zr}$ in the present work.

\begin{tabular}{lccccc}
\hline \hline$E_{i}(\mathrm{keV})$ & $E_{\gamma}(\mathrm{keV})$ & $B(E 2)(\mathrm{W} . \mathrm{u})$. & $\mathrm{Q}_{t}(\mathrm{eb})$ & $\beta_{2}$ & Band \\
\hline 821.6 & 143.0 & $99(6)$ & $3.3(1)$ & $0.34(1)$ & $\nu 3 / 2[541]$ \\
1236.6 & 415.0 & $66(5)$ & $2.4(3)$ & $0.26(3)$ & $\nu 3 / 2[541]$ \\
850.5 & 274.5 & $46(12)$ & $2.9(3)$ & $0.32(3)$ & $v 3 / 2[411]$ \\
\hline \hline
\end{tabular}




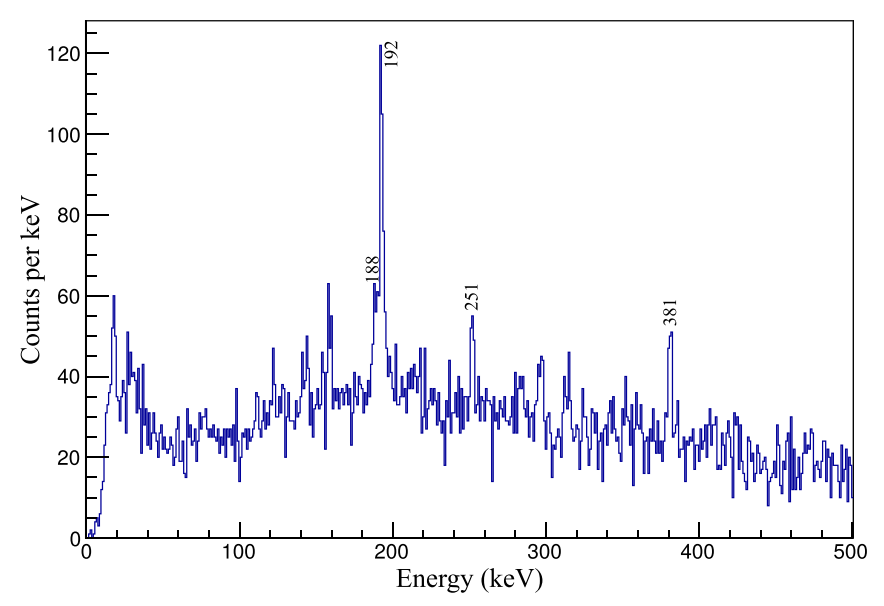

FIG. 8. Energy spectra obtained when gating on the $121.7-\mathrm{keV}$ $\left[\mathrm{Ge} / \mathrm{LaBr}_{3}(\mathrm{Ce})\right], 536.0-\mathrm{keV}(\mathrm{Ge})$, and 218.0-keV [Ge/LaBr $\left.3(\mathrm{Ce})\right]$ $\gamma$ rays.

575.7-keV level was not reported [27]. The mixed nature of this band was proposed due to the poor agreement between $B(M 1) / B(E 2)$ values derived from $\frac{\Delta J=1}{\Delta J=2}$ branching ratios and theoretically predicted ones [27].

The $381-\mathrm{keV}$ transition was rejected in the evaluation by [31] as it is inconsistent with the adopted $J^{\pi}=3 / 2^{+}$assigned to the $657.9-\mathrm{keV}$ level. Figure 8 shows a coincident $\gamma$-ray spectrum. This figure corresponds to a gate on the 218-keV transition, which feeds the $K=9 / 2^{+}$isomer, and gates on the 536.0- and 121.7-keV transitions, which correspond to the cascade of decays from the $657.9-\mathrm{keV}$ level. The $381-\mathrm{keV}$ transition is clearly present along with the $251-\mathrm{keV}$ transition that feeds the $J^{\pi}=11 / 2^{+}$above the $K=9 / 2^{+}$isomer. Therefore a $J^{\pi}=3 / 2^{+}$assignment for the $657.9-\mathrm{keV}$ level is rejected and the $J^{\pi}=5 / 2^{+}$assignment as suggested in Ref. [18] is proposed to be correct. The disparity in intensity between the $192.6-$ and $188.5-\mathrm{keV}$ transitions is due to the 215.5-keV transition which, populating the $850.5-\mathrm{keV}$ level, has an energy similar to the $218-\mathrm{keV}$ coincidence gate.

The reduced $M 1$ transition rates obtained in the present work, $B(M 1)=0.032(10)$ and $0.042(21)$ W.u. for the 192.6and $82.2-\mathrm{keV}$ transitions, respectively, are the same, within errors. This implies the 850.5-, 657.9-, and 575.7-keV levels have a similar structure, or are all members of the same band, in agreement with [18]. The low intensity of the 82.2-keV intraband transition is therefore a consequence of other strong decay paths out of the $657.9-\mathrm{keV}$ level. Its energy is likely perturbed by interactions with other nearby $\left(3 / 2^{+}\right)$states, or the $3 / 2_{1}^{+}$level.

The band containing the 575.7-, 657.9-, and $850.5-\mathrm{keV}$ levels has been proposed to have a $v 3 / 2[411]$ configuration, which has a significant $\nu g_{7 / 2}$ component [18]. The $\log f t$ values derived for $\beta$ feeding to these levels are 6.3, 6.7, and 6.6, respectively [40]. These values are much higher than those of an allowed Gamow-Teller transition, likely due to the strong spherical $v d_{5 / 2}$ single-particle component and quasihole nature of the $v 3 / 2[411]$ orbital. The collectivity present in the band will also fragment the $v g_{7 / 2}$ strength across several levels, resulting in higher $\log f t$ values. The

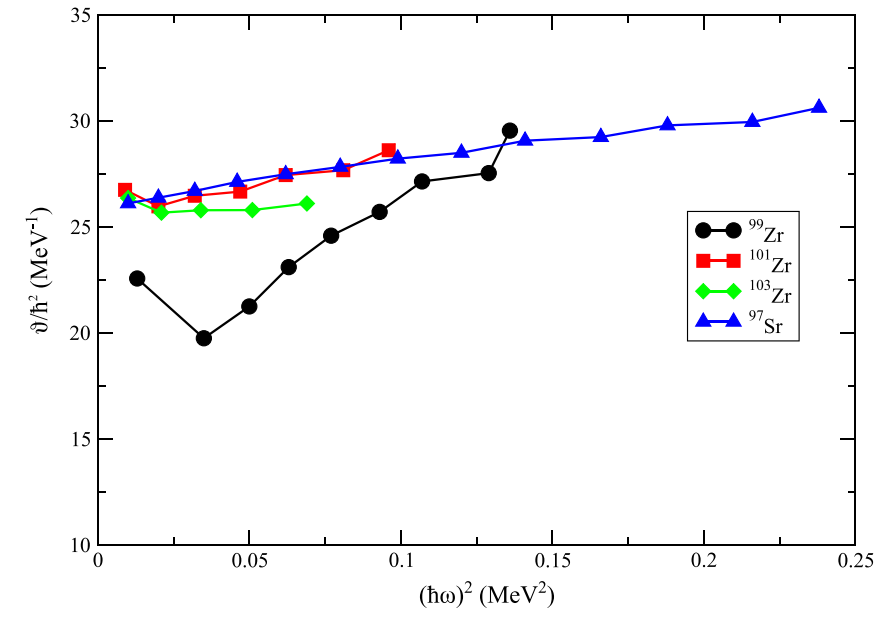

FIG. 9. Kinematic moments of inertia of v3/2[411] bands in ${ }^{99,101,103} \mathrm{Zr}$ and ${ }^{97} \mathrm{Sr}$.

shape coexistence present in ${ }^{99} \mathrm{Zr}$ and mixed configurations means that it is unclear if asymptotic Nilsson orbital labels are applicable. Nevertheless, a v3/2[411] label is kept in the article for consistency with the existing literature.

\section{B. $v 3 / 2[541]$ band}

The lifetimes of the three lowest lying members of the v3/2[541] band, based on the 614.1-keV level were previously measured [40]. These levels were populated following the $\beta$ decay of ${ }^{99} \mathrm{Y}$ parent nuclei, though only one $B(E 2)$ value was obtained, $B\left(E 2 ; 7 / 2^{-} \rightarrow 3 / 2^{-}\right)=210(70)$ W.u.

The values of $\beta_{2}=0.34(1)$ and $0.26(3)$ derived for the $\left(11 / 2^{-}\right)$and $\left(15 / 2^{-}\right)$members of the same $v 3 / 2[541]$ band of ${ }^{99} \mathrm{Zr}$ in the present work are inconsistent. The latter $\beta_{2}$ value, however, agrees with $\beta_{2}=0.28(1)$ measured for this band's $\left(19 / 2^{-}\right)$and $\left(23 / 2^{-}\right)$members [17]. It is also close to $\beta_{2}=$ 0.32 (2) determined for the same band in the isotone ${ }^{97} \mathrm{Sr}$ [17]. The change in quadrupole moment across the $821.6-\mathrm{keV}$ state, with spin $\left(11 / 2^{-}\right)$, may indicate a crossing with a Nilsson orbital of the same spin. A deformation of $\beta_{2}=0.356(8)$ has been reported for the $\left(2^{+}\right)$state of ${ }^{100} \mathrm{Zr}$ [41], showing that the collectivities of members of the v3/2[541] band are close to that of the deformed even-even ground-state core. Simplistically this band can be thought of as a v3/2[541] hole coupled to a ${ }^{100} \mathrm{Zr}$ core, with the hole acting to reduce the quadrupole moment.

\section{Moments of inertia}

Differences in the kinematic moment of inertia of the $v 3 / 2$ [411] band in ${ }^{99} \mathrm{Zr}$, compared to other bands of this nucleus have been noted by Wu et al. [27]. This quantity is plotted for the $v 3 / 2[411]$ bands of ${ }^{99,101,103} \mathrm{Zr}$ and ${ }^{97} \mathrm{Sr}$ in Fig. 9. The $\nu 3 / 2$ [411] bands of ${ }^{101,103} \mathrm{Zr}$ and ${ }^{97} \mathrm{Sr}$ have almost constant moments of inertia, indicating rigid rotation, whereas the slope for the $v 3 / 2$ [411] band of ${ }^{99} \mathrm{Zr}$ indicates a degree of softness in the core. Figure 10 of Wu et al. [27] shows that the moments of inertia of the 3/2[541] bands of ${ }^{97} \mathrm{Sr}$ and ${ }^{99} \mathrm{Zr}$ are also consistent with rigid rotation, with values similar to 


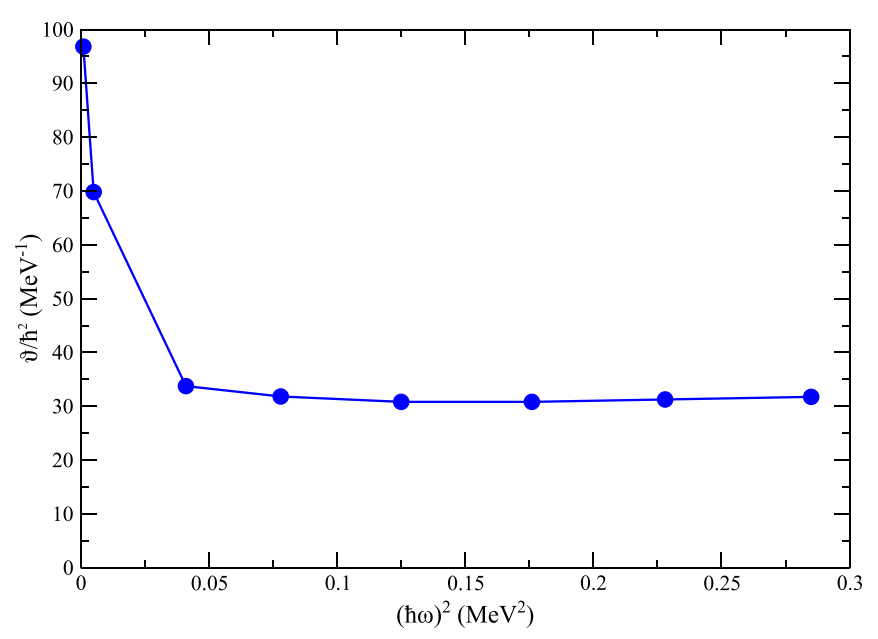

FIG. 10. Kinematic moments of inertia of $v 3 / 2$ [451] bands in ${ }^{99} \mathrm{Zr}$.

those of the $v 3 / 2$ [411] bands of ${ }^{97} \mathrm{Sr}$ and ${ }^{101,103} \mathrm{Zr}$. Despite being at similar energies, the $v 3 / 2[411]$ and $v 3 / 2$ [541] bands of ${ }^{99} \mathrm{Zr}$ behave differently, and it seems that the occupation of specific orbitals in the underlying core provokes a structural change in the core and affects the rotational properties. The $E\left(4_{1}^{+}\right) / E\left(2_{1}^{+}\right)$ratio of ${ }^{100} \mathrm{Zr}$ is 2.66 , characteristic of a transitional or $\gamma$-unstable nucleus, whereas those of ${ }^{98} \mathrm{Sr}$ and ${ }^{102} \mathrm{Zr}$ are 3.00 and 3.15 , respectively, close to the rigid-rotor limit.

The soft nature of the $v 3 / 2$ [411] band in ${ }^{99} \mathrm{Zr}$ provides evidence that occupation of the $\nu g_{7 / 2}$ and/or $v d_{5 / 2}$ orbitals does not reduce the pairing correlations present in the core as much as in the bands of ${ }^{101,103} \mathrm{Zr}$. The stiffer nature of the core and larger deformation of the $v 3 / 2[541]$ band points towards occupation of the $v h_{11 / 2}$ orbital in reducing the pairing correlations present in the core and a move towards a rigid, well-deformed rotor regime. Differences in the kinematic moment of inertia of the $v 3 / 2$ [451] band in ${ }^{99} \mathrm{Zr}$ are presented in Fig. 10. The band has a near constant moment of inertia with the exception of the low-spin levels, which coincide with the large increase in $B(E 2)$ values. This indicates that rigid rotation is is not a suitable description for the low-spin levels within the $v 3 / 2[451]$ band.

\section{IBFM-1}

As a result of the recent measurement [42] of the magnetic moment, $\mu$, of the $7 / 2_{1}^{+}$state of ${ }^{99} \mathrm{Zr}$, the three lowest-lying states of this nucleus now have a rather complete experimental characterization, with experimentally determined magnetic moments and electromagnetic transition rates [17,18,27]. Since the lowest-lying states in an odd-mass nucleus are mainly of one-quasiparticle nature, it was decided to investigate their description with a simple model, the interacting boson-fermion model (IBFM) in its version IBFM-1, which does not distinguish between protons and neutrons [43,44]. In this model, the odd nucleus is described by coupling an
TABLE IV. Values for the single-particle energies $\epsilon_{j}$, their respective occupation probabilities $V_{j}^{2}$, and quasiparticle energies $E_{j}$ of the orbitals used in the IBFM-1 calculations for ${ }^{99} \mathrm{Zr}$.

\begin{tabular}{lccc}
\hline \hline Orbital & $\epsilon_{j}(\mathrm{MeV})$ & $V_{j}^{2}$ & $E_{j}(\mathrm{MeV})$ \\
\hline$v d_{5 / 2}$ & 0.4 & 0.5437 & 1.506 \\
$\nu g_{7 / 2}$ & 1.9 & 0.1630 & 2.030 \\
$\nu s_{1 / 2}$ & 0.0 & 0.6671 & 1.591 \\
$\nu d_{3 / 2}$ & 0.7 & 0.4443 & 1.509 \\
$\nu h_{11 / 2}$ & 2.4 & 0.1101 & 2.396 \\
\hline \hline
\end{tabular}

odd nucleon, allowed to occupy single-particle shell-model orbitals, to an even-even core described by the interaction boson model 1 (IBM-1) [45,46]. The description of the known spectroscopic properties of the lowest-lying states allowed a fine tuning of the IBFM-1 parameters [42] (see also Table IV). Here we use the results of these calculations to investigate their ability to describe the properties of other excited states, including those determined in this experiment.

It should, however, be emphasized that the IBFM-1 description has strong limitations. Nuclei in this region of rapid shape transition, around $N=60$, have been found to exhibit shape coexistence phenomena, which cannot be accounted for by this model. The fast structure evolution around $N=60$ also makes it difficult to choose an appropriate even-even core nucleus for ${ }^{99} \mathrm{Zr}$ as the ground-state deformation changes drastically from ${ }^{98} \mathrm{Zr}$ to ${ }^{100} \mathrm{Zr}$, its two even-even neighbors. Here, ${ }^{100} \mathrm{Zr}$ was chosen as a core due to the similarity between the energies of its ground-state band members and the energy distribution of the low-lying states in ${ }^{99} \mathrm{Zr}$. The IBM-1 description employed for the core was taken from Ref [47]. The odd neutron coupled to the core was allowed to span the single-particle orbits of the $N=50-82$ valence space $\left(s_{1 / 2}, d_{3 / 2}, d_{5 / 2}, g_{7 / 2}\right.$, and $\left.h_{11 / 2}\right)$. The IBFM-1 calculations are similar to those performed in Ref. [48] for the isotonic nucleus ${ }^{97} \mathrm{Sr}$, which presents structure similarities with ${ }^{99} \mathrm{Zr}$. The main difference from the calculations in Ref. [48] is the inclusion of the $v h_{11 / 2}$ orbital in the present calculations, and the use of the same IBFM-1 parameters for the description of both positive and negative parity states.

The single-particle energies for nuclei in this region are not known; they were adjusted to reasonably reproduce the low-energy part of the experimental level scheme. The values adopted for these calculations are given in Table IV, together with quasiparticle energies obtained from BCS calculations with a pairing parameter $\Delta=1.5 \mathrm{MeV}$. The values chosen for the boson-fermion interaction parameters $[43,44]$ were $A_{0}=0.08 \mathrm{MeV}, \Gamma_{0}=0.3 \mathrm{MeV}, \Lambda_{0}=2.9 \mathrm{MeV}^{2}$, and $\hbar \omega=$ $1.5 \mathrm{MeV}$.

Electromagnetic $E 2$ transition rates were calculated using a boson charge $e_{B}=0.159 \mathrm{eb}$ which describes the $B(E 2)$ values in ${ }^{100} \mathrm{Zr}$ [47] and a fermion charge $e_{F}$ with the same value. For the $M 1$ transitions we used the simplest transfer operator, with gyromagnetic ratios $g_{d}=0.4 \mu_{N}$ for the $d$-boson $g$ factor, and $g_{s}=-2.68 \mu_{N}$ for the fermion, which is $70 \%$ of the free neutron value to account for core polarization effects, and a value $g_{l}=0$ (the odd nucleon is a neutron). 


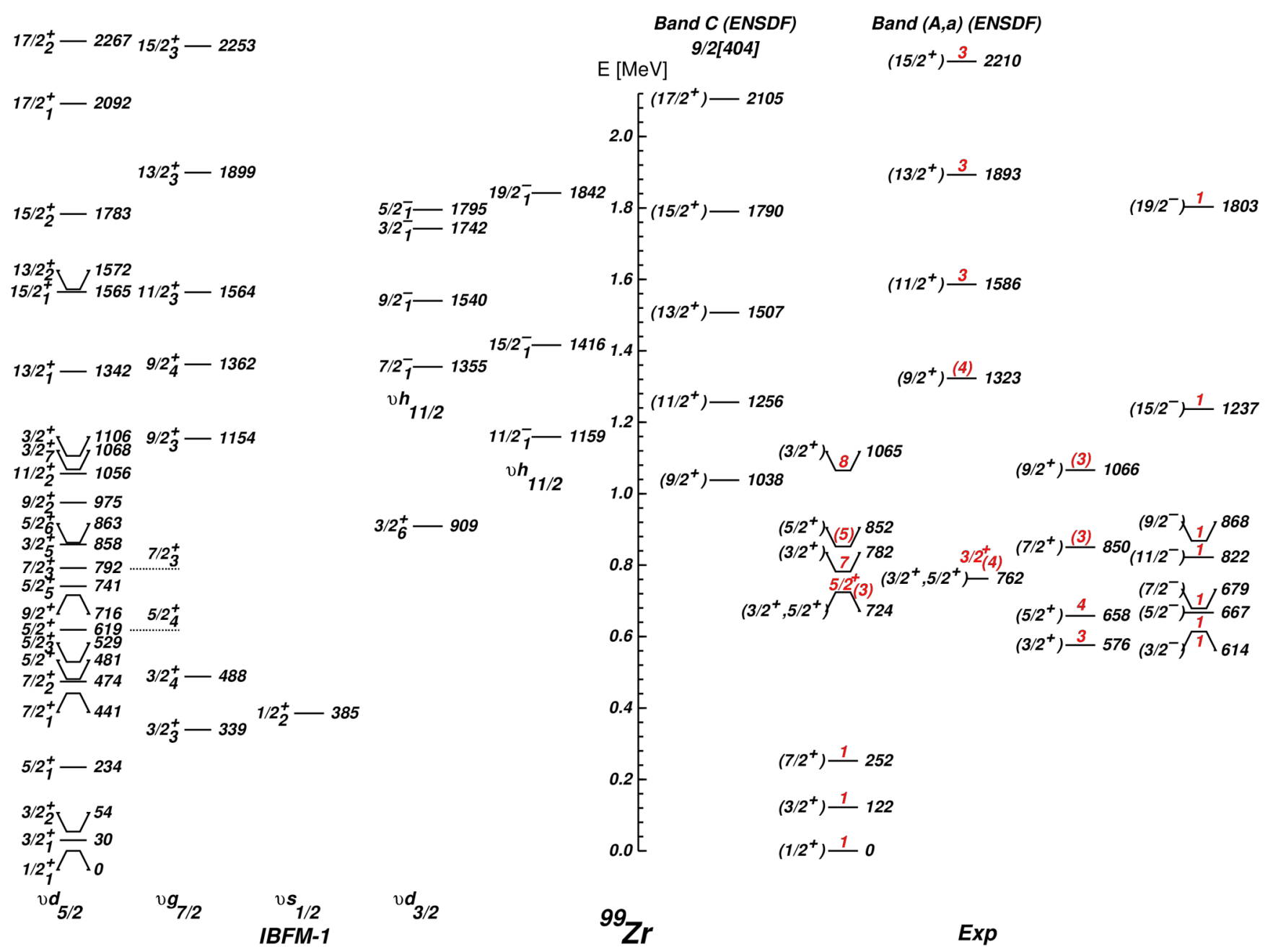

FIG. 11. Calculated and experimental level schemes of ${ }^{99} \mathrm{Zr}$. Experimental states are labeled with a number corresponding to the order number of their theoretical counterpart for a given spin. For tentative assignments this number is placed within parentheses.

Figure 11 shows a comparison of the calculated level scheme with the experimental one. The theoretical levels (left side of the figure) are arranged according to the dominating quasiparticle component in their wave functions, with the observation that a general feature is the rather strong mixing between the positive parity orbitals, especially the $d_{5 / 2}$ and $g_{7 / 2}$ ones. The experimental level scheme (right side of the figure) highlights some differences between the ENSDF adopted level scheme [31] and the spin assignments of Ref. [18] (see also Fig. 1), showing two distinct bandlike structures: one based on the $576-\mathrm{keV} 3 / 2^{+}$state, and the remaining part of the $(A, a)$ structure above the $1323-\mathrm{keV} 9 / 2^{+}$state.

In Fig. 11 correspondences between calculated and experimental levels are indicated. These are based not only on the excitation energy, but also on the description of the experimental $\gamma$-decay patterns of the states (branching ratios and, when known, reduced transition probabilities). The correspondence between calculated and experimental levels is indicated by the order number of the calculated states of each spin. Tentative associations are indicated by placing this order number within parentheses.
For positive parities, besides the lowest energy states, a one-to-one correspondence with calculated states could be established in this way for a number of states with spin $3 / 2$ and $5 / 2$ in the energy range $\approx 600-1000 \mathrm{keV}$ Also, for the higherspin members of the $(A, a)$ structure, theoretical counterparts have been proposed. Some difficulties were encountered in the description of the decay patterns of the states at 850, 1066, and $1323 \mathrm{keV}$, therefore their theoretical assignment is only tentative. Table V shows the comparison between calculated and experimental transition rates. While for the lower-energy states the agreement is good, for the $850-\mathrm{keV} 7 / 2^{+}$state it is worse, especially its $E 2$ branch towards the $576-\mathrm{keV} 3 / 2^{+}$ state is predicted less collective, with a $B(E 2)$ value 3-4 times smaller than the experimental value. The decay pattern of the $1323-\mathrm{keV} 9 / 2^{+}$state towards the $850-\mathrm{keV} 7 / 2^{+}$and $1066-\mathrm{keV} 9 / 2^{+}$states [31] also could not be well reproduced (therefore, these states are only tentatively associated with calculated ones). It appears that the connection between the two level structures [band $(A, a)$ and the band based on the $576-\mathrm{keV} 3 / 2^{+}$state[ cannot be well described by this simple model (in terms of these calculations, one would need a 
TABLE V. Level energies $E_{i}$, transition energies $E_{\gamma}$, and experimental and theoretical reduced transition rates for states of ${ }^{99} \mathrm{Zr}$ relevant to the present work. Spin assignments correspond to values given in Fig. 11. All $\Delta J=0,1$ transitions were considered to be pure $M 1$ in the experimental analysis.

\begin{tabular}{|c|c|c|c|c|c|c|c|}
\hline \multirow{2}{*}{$\begin{array}{l}E_{i} \\
(\mathrm{keV})\end{array}$} & \multirow{2}{*}{$\begin{array}{c}E_{\gamma} \\
(\mathrm{keV})\end{array}$} & \multirow[t]{2}{*}{$J_{i}$} & \multirow[t]{2}{*}{$J_{f}$} & \multicolumn{2}{|c|}{$B(E 2)$ (W.u.) } & \multicolumn{2}{|c|}{$B(M 1)$ (W.u.) } \\
\hline & & & & exp. & calc. & exp. & calc. \\
\hline 121.7 & 121.7 & $3 / 2^{+}$ & $1 / 2^{+}$ & & & $0.0101(7)$ & 0.0109 \\
\hline \multirow[t]{2}{*}{657.9} & 82.2 & $5 / 2^{+}$ & $3 / 2^{+}$ & & & $0.042(21)$ & 0.078 \\
\hline & 536.0 & $5 / 2^{+}$ & $3 / 2^{+}$ & & & $0.0047(20)$ & 0.0064 \\
\hline \multirow[t]{2}{*}{850.5} & 192.6 & $7 / 2^{+}$ & $5 / 2^{+}$ & & & $0.032(10)$ & 0.096 \\
\hline & 274.5 & $7 / 2^{+}$ & $3 / 2^{+}$ & $46(12)$ & 9.5 & & \\
\hline 821.6 & 143.0 & $11 / 2^{-}$ & $7 / 2^{-}$ & $99(6)$ & 81.1 & & \\
\hline 1236.6 & 415.0 & $15 / 2^{-}$ & $11 / 2^{-}$ & $60(11)$ & 99 & & \\
\hline 1802.6 & 566.0 & $19 / 2^{-}$ & $15 / 2^{-}$ & $66(9)^{\mathrm{a}}$ & 118 & & \\
\hline
\end{tabular}

aTaken from Ref. [17].

different mixing of the $d_{5 / 2}$ and $g_{7 / 2}$ orbitals in this energy region). The experimental band labeled "Band C" in Fig. 11, which was firmly assigned as $v 9 / 2$ [404] band originating from the $g_{9 / 2}$ orbital [31], cannot be described with the present approach.

For the negative parity states, only the fully aligned sequence of states $11 / 2^{-}, 15 / 2^{-}$, and $19 / 2^{-}$are reasonably described, while the states of lower spins are predicted to be higher in energy. This may be due to a number of factors, such as the considerable change of structure around $N=60$, or a drastic change in the monopole energy of the $v h_{11 / 2}$ orbital.

\section{E. Appearance of collectivity in ${ }^{99} \mathrm{Zr}$}

The reasonable reproduction of the level scheme of ${ }^{99} \mathrm{Zr}$ by the IBFM-1 calculations allows some conclusions concerning the onset of collective behavior in this nucleus. Collective modes occur when the integrated proton-neutron interaction overcomes the spherical symmetry restoring pairing force. Quantitatively this happens when $\epsilon-\Delta<\lambda$, where $\lambda$ is the energy of the Fermi surface. The BCS calculation in Sec. IV D gives $\lambda=0.327 \mathrm{MeV}$ using $\Delta_{N}=1.5 \mathrm{MeV}$. Examining the single-particle energies $\epsilon$ in Table IV shows that the $v s_{1 / 2}$, $v d_{3 / 2}$, and $v d_{5 / 2}$ orbits fulfill the condition for a collective regime whereas $\epsilon-\Delta \approx \lambda$ for the $\nu g_{7 / 2}$ orbit. These four orbits form a pseudo-SU(3) algebraic group, corresponding to a deformed rotor. One notes that the $\Delta L=\Delta J=2 s_{1 / 2}-d_{5 / 2}$ and $d_{3 / 2}-g_{7 / 2}$ couplings are quasi-SU(3) blocks.

From Table IV one can see that the four positive-parity orbitals chosen in this work become almost degenerate in ${ }^{99} \mathrm{Zr}$, after following a relatively smooth evolution with the number of neutrons in the isotopes from ${ }^{91} \mathrm{Zr}$ to ${ }^{97} \mathrm{Zr}$ (experimental data from [49]). This behavior is similar to that found for the isotonic nucleus ${ }^{97} \mathrm{Sr}[10]$. The near-degenerate nature of the four neutron quasiparticle energies listed would not be expected to generate the strong collectivity present towards the bottom of the $v 3 / 2[541]$ and $v 3 / 2$ [411] bands. Multiquasiparticle bands are expected at an energy of $\sim 2 \Delta_{N}$, much higher than the bandhead energies. Another mechanism must be present which significantly changes the nuclear structure, most likely type-II shell evolution. In particular, the poor agreement between the calculated and experimental members of the $v 3 / 2$ [541] band may be due to the monopole evolution of the $v h_{11 / 2}$ orbit, known to be shifted in energy by type-II shell evolution [25].

As observed above, with $N$ increasing from $51\left({ }^{91} \mathrm{Zr}\right)$ to $57\left({ }^{97} \mathrm{Zr}\right)$, the single-particle energies of the positive-parity orbitals evolve rather smoothly as the $v d_{5 / 2}$ and $v s_{1 / 2}$ orbits are filled, while the energy of the $\nu h_{11 / 2}$ orbit increases. The $v h_{11 / 2}$ orbit rises in energy with increasing $v d_{5 / 2}$ occupation due to the repulsive nature of the tensor force for two alike $l+s$ orbits. At $N=59$ a drastic change in the single-particle energies occurs, with the overall separation of all orbits greatly reduced. This coincides with significant occupation of $\pi g_{9 / 2}, v g_{7 / 2}$, and $v h_{11 / 2}$ orbits, providing evidence that monopole evolution occurs in collective regions.

\section{CONCLUSION}

Lifetimes of states in the $v 3 / 2[541]$ and $v 3 / 2[411]$ rotational bands of the nucleus ${ }^{99} \mathrm{Zr}$ have been measured using the fast-timing technique. The ${ }^{99} \mathrm{Zr}$ nuclei were populated following the neutron-induced fission of a ${ }^{235} \mathrm{U}$ target during the EXILL-FATIMA campaign. The v3/2[541] band was deduced to possess quadrupole deformations of $\beta_{2}=0.34(1)$ and $0.26(3)$ at spins of $\left(11 / 2^{-}\right)$and $\left(15 / 2^{-}\right)$, whereas the $\left(7 / 2^{+}\right)$member of the $3 / 2[411]$ band has $\beta_{2}=0.32(3)$. IBFM-1 calculations reasonably reproduce characteristics of many of the known levels of ${ }^{99} \mathrm{Zr}$. An exception are the energies of the "antialigned" levels (of spin lower that 11/2) of the negative parity band, which in these calculations has a pure $v h_{11 / 2}$ origin. Type-II shell evolution is proposed to play a major role in the creation of low-lying rotational bands of ${ }^{99} \mathrm{Zr}$.

\section{ACKNOWLEDGMENTS}

This work was supported by NuPNET and the German BMBF by Grants No. 05P12PKNUF, No. 05P15RDFN1 and No. 05P12DRNUP, the German Research Council (DFG) under Grant No. SFB 1245, the Spanish MINECO via Project No. FPA2015-65035-P, the UK STFC by Contract No. DNC7RP01/4, the US DOE under Grant No. DE-FG0291ER40609, and the UK National Measurement Office. The EXILL-FATIMA campaign would not have been possible without the support of several services at the ILL and the LPSC. We are grateful to the EXOGAM Collaboration for the loan of the detectors, to GANIL for assistance during installation and dismantling, and to the FATIMA Collaboration for the provision of $\mathrm{LaBr}_{3}(\mathrm{Ce})$ detectors and analog electronics.
[1] H. L. Thayer, J. Billowes, P. Campbell, P. Dendooven, K. T. Flanagan, D. H. Forest, J. A. R. Griffith, J. Huikari, A. Jokinen,
R. Moore, A. Nieminen, G. Tungate, S. Zemlyanoi, and J. Äystö, J. Phys. G: Nucl. Part. Phys. 29, 2247 (2003). 
[2] E. Cheifetz, R. C. Jared, S. G. Thompson, and J. B. Wilhelmy, Phys. Rev. Lett. 25, 38 (1970).

[3] K. Shizuma, H. Lawin, and K. Sistemich, Z. Phys. A 311, 71 (1983).

[4] P. Bonche, H. Flocard, P. H. Heenen, S. J. Krieger, and M. S. Weiss, Nucl. Phys. A 443, 39 (1985).

[5] R. Rodríguez-Guzmán, P. Sarriguren, L. Robledo, and S. PerezMartin, Phys. Lett. B 691, 202 (2010).

[6] T. W. Hagen, A. Görgen, W. Korten, L. Grente, M. D Salsac, F. Farget, T. Braunroth, B. Bruyneel, I. Celikovic, E. Clément, G. de France, O. Delaune, A. Dewald, A. Dijon, M. Hackstein, B. Jacquot, J. Litzinger, J. Ljungvall, C. Louchart, C. Michelagnoli, D. R. Napoli, F. Recchia, W. Rother, E. Sahin, S. Siem, B. Sulignano, C. Theisen, and J. J. Valiente-Dobon, Eur. Phys. J. A 54, 50 (2018).

[7] M. Albers, N. Warr, K. Nomura, A. Blazhev, J. Jolie, D. Mücher, B. Bastin, C. Bauer, C. Bernards, L. Bettermann, V. Bildstein, J. Butterworth, M. Cappellazzo, J. Cederkäll, D. Cline, I. Darby, S. Das Gupta, J. M. Daugas, T. Davinson, H. De Witte, J. Diriken, D. Filipescu, E. Fiori, C. Fransen, L. P. Gaffney, G. Georgiev, R. Gernhäuser, M. Hackstein, S. Heinze, H. Hess, M. Huyse, D. Jenkins, J. Konki, M. Kowalczyk, T. Kröll, R. Krücken, J. Litzinger, R. Lutter, N. Marginean, C. Mihai, K. Moschner, P. Napiorkowski, B. S. Nara Singh, K. Nowak, T. Otsuka, J. Pakarinen, M. Pfeiffer, D. Radeck, P. Reiter, S. Rigby, L. M. Robledo, R. Rodríguez-Guzmán, M. Rudigier, P. Sarriguren, M. Scheck, M. Seidlitz, B. Siebeck, G. Simpson, P. Thöle, T. Thomas, J. Van de Walle, P. Van Duppen, M. Vermeulen, D. Voulot, R. Wadsworth, F. Wenander, K. Wimmer, K. O. Zell, and M. Zielinska, Phys. Rev. Lett. 108, 062701 (2012).

[8] F. Flavigny, P. Doornenbal, A. Obertelli, J.-P. Delaroche, M. Girod, J. Libert, T. R. Rodriguez, G. Authelet, H. Baba, D. Calvet, F. Château, S. Chen, A. Corsi, A. Delbart, J.-M. Gheller, A. Giganon, A. Gillibert, V. Lapoux, T. Motobayashi, M. Niikura, N. Paul, J.-Y. Roussé, H. Sakurai, C. Santamaria, D. Steppenbeck, R. Taniuchi, T. Uesaka, T. Ando, T. Arici, A. Blazhev, F. Browne, A. Bruce, R. Carroll, L. X. Chung, M. L. Cortés, M. Dewald, B. Ding, S. Franchoo, M. Górska, A. Gottardo, A. Jungclaus, J. Lee, M. Lettmann, B. D. Linh, J. Liu, Z. Liu, C. Lizarazo, S. Momiyama, K. Moschner, S. Nagamine, N. Nakatsuka, C. Nita, C. R. Nobs, L. Olivier, R. Orlandi, Z. Patel, Z. Podolyák, M. Rudigier, T. Saito, C. Shand, P. A. Söderström, I. Stefan, V. Vaquero, V. Werner, K. Wimmer, and Z. Xu, Phys. Rev. Lett. 118, 242501 (2017).

[9] R. Sheline, I. Ragnarsson, and S. Nilsson, Phys. Lett. B 41, 115 (1972).

[10] G. Lhersonneau, B. Pfeiffer, K.-L. Kratz, T. Enqvist, P. P. Jauho, A. Jokinen, J. Kantele, M. Leino, J. M. Parmonen, H. Penttilä, and J. Äystö (ISOLDE Collaboration), Phys. Rev. C 49, 1379 (1994).

[11] M. Büscher, R. F. Casten, R. L. Gill, R. Schuhmann, J. A. Winger, H. Mach, M. Moszyński, and K. Sistemich, Phys. Rev. C 41, 1115 (1990).

[12] E. Clément, M. Zielińska, A. Görgen, W. Korten, S. Péru, J. Libert, H. Goutte, S. Hilaire, B. Bastin, C. Bauer, A. Blazhev, N. Bree, B. Bruyneel, P. A. Butler, J. Butterworth, P. Delahaye, A. Dijon, D. T. Doherty, A. Ekström, C. Fitzpatrick, C. Fransen, G. Georgiev, R. Gernhäuser, H. Hess, J. Iwanicki, D. G. Jenkins, A. C. Larsen, J. Ljungvall, R. Lutter, P. Marley, K. Moschner, P. J. Napiorkowski, J. Pakarinen, A. Petts, P. Reiter,
T. Renstrøm, M. Seidlitz, B. Siebeck, S. Siem, C. Sotty, J. Srebrny, I. Stefanescu, G. M. Tveten, J. Van de Walle, M. Vermeulen, D. Voulot, N. Warr, F. Wenander, A. Wiens, H. De Witte, and K. Wrzosek-Lipska, Phys. Rev. Lett. 116, 022701 (2016)

[13] H. Mach, F. K. Wohn, M. Moszyn'ski, R. L. Gill, and R. F. Casten, Phys. Rev. C 41, 1141 (1990).

[14] Å. W. Iskra, B. Fornal, S. Leoni, G. Bocchi, A. Petrovici, C. Porzio, A. Blanc, G. D. France, M. Jentschel, U. Köster, P. Mutti, J.-M. Régis, G. Simpson, T. Soldner, C. A. Ur, W. Urban, D. Bazzacco, G. Benzoni, S. Bottoni, A. Bruce, N. CieplickaOryńczak, F. C. L. Crespi, L. M. Fraile, W. Korten, T. Kröll, S. Lalkovski, N. Mărginean, C. Michelagnoli, B. Melon, D. Mengoni, B. Million, A. Nannini, D. Napoli, Z. Podolyák, P. H. Regan, and B. Szpak, Europhys. Lett. 117, 12001 (2017).

[15] B. Cheal, M. Gardner, M. Avgoulea, J. Billowes, M. Bissell, P. Campbell, T. Eronen, K. Flanagan, D. Forest, J. Huikari, A. Jokinen, B. Marsh, I. Moore, A. Nieminen, H. Penttilä, S. RintaAntila, B. Tordoff, G. Tungate, and J. Äystö, Phys. Lett. B 645, 133 (2007).

[16] W. Witt, V. Werner, N. Pietralla, M. Albers, A. D. Ayangeakaa, B. Bucher, M. P. Carpenter, D. Cline, H. M. David, A. Hayes, C. Hoffman, R. V. F. Janssens, B. P. Kay, F. G. Kondev, W. Korten, T. Lauritsen, O. Möller, G. Rainovski, G. Savard, D. Seweryniak, J. Smith, R. Stegmann, S. Zhu, and C. Y. Wu, Phys. Rev. C 98, 041302(R) (2018).

[17] W. Urban, J. Durell, A. Smith, W. Phillips, M. Jones, B. Varley, T. Rząca-Urban, I. Ahmad, L. Morss, M. Bentaleb, and N. Schulz, Nucl. Phys. A 689, 605 (2001).

[18] W. Urban, J. Pinston, T. Rzaca-Urban, A. Złomaniec, G. Simpson, J. Durell, W. Phillips, A. Smith, B. Varley, I. Ahmad, and N. Schulz, Eur. Phys. J. A 16, 11 (2003).

[19] C. Kremer, S. Aslanidou, S. Bassauer, M. Hilcker, A. Krugmann, P. von Neumann-Cosel, T. Otsuka, N. Pietralla, V. Y. Ponomarev, N. Shimizu, M. Singer, G. Steinhilber, T. Togashi, Y. Tsunoda, V. Werner, and M. Zweidinger, Phys. Rev. Lett. 117, 172503 (2016).

[20] P. Federman and S. Pittel, Phys. Lett. B 69, 385 (1977).

[21] P. Federman and S. Pittel, Phys. Lett. B 77, 29 (1978).

[22] P. Federman and S. Pittel, Phys. Rev. C 20, 820 (1979).

[23] P. Cejnar, J. Jolie, and R. F. Casten, Rev. Mod. Phys. 82, 2155 (2010).

[24] T. Otsuka and Y. Tsunoda, J. Phys. G: Nucl. Part. Phys. 43, 024009 (2016).

[25] T. Togashi, Y. Tsunoda, T. Otsuka, and N. Shimizu, Phys. Rev. Lett. 117, 172502 (2016).

[26] K. Heyde and J. L. Wood, Rev. Mod. Phys. 83, 1467 (2011).

[27] C. Y. Wu, H. Hua, D. Cline, A. B. Hayes, R. Teng, R. M. Clark, P. Fallon, A. Goergen, A. O. Macchiavelli, and K. Vetter, Phys. Rev. C 70, 064312 (2004).

[28] H. Abele, D. Dubbers, H. Häse, M. Klein, A. Knöpfler, M. Kreuz, T. Lauer, B. Märkisch, D. Mund, V. Nesvizhevsky, A. Petoukhov, C. Schmidt, M. Schumann, and T. Soldner, Nucl. Instrum. Methods Phys. Res., Sect. A 562, 407 (2006).

[29] J.-M. Régis, G. Simpson, A. Blanc, G. de France, M. Jentschel, U. Köster, P. Mutti, V. Paziy, N. Saed-Samii, T. Soldner, C. Ur, W. Urban, A. Bruce, F. Drouet, L. Fraile, S. Ilieva, J. Jolie, W. Korten, T. Kröll, S. Lalkovski, H. Mach, N. Mărginean, G. Pascovici, Z. Podolyak, P. Regan, O. Roberts, J. Smith, C. Townsley, A. Vancraeyenest, and N. Warr, Nucl. Instrum. Methods Phys. Res., Sect. A 763, 210 (2014). 
[30] W. Urban, M. Jentschel, B. Markisch, T. Materna, C. Bernards, C. Drescher, C. Fransen, J. Jolie, U. Köster, P. Mutti, T. RzącaUrban, and G. Simpson, J. Instrum. 8, P03014 (2013).

[31] E. Browne and J. Tuli, Nucl. Data Sheets 145, 25 (2017).

[32] P. Spagnoletti, G. S. Simpson, R. Carroll, J.-M. Régis, A. Blanc, M. Jentschel, U. Köster, P. Mutti, T. Soldner, G. de France, C. A. Ur, W. Urban, A. M. Bruce, F. Drouet, L. M. Fraile, L. P. Gaffney, D. G. Ghită, S. Ilieva, J. Jolie, W. Korten, T. Kröll, C. Larijani, S. Lalkovski, R. Lică, H. Mach, N. Mărginean, V. Paziy, Z. Podolyák, P. H. Regan, M. Scheck, N. Saed-Samii, G. Thiamova, C. Townsley, A. Vancraeyenest, V. Vedia, A. Gargano, and P. Van Isacker, Phys. Rev. C 95, 021302(R) (2017).

[33] S. Ansari, J.-M. Régis, J. Jolie, N. Saed-Samii, N. Warr, W. Korten, M. Zielińska, M.-D. Salsac, A. Blanc, M. Jentschel, U. Köster, P. Mutti, T. Soldner, G. S. Simpson, F. Drouet, A. Vancraeyenest, G. de France, E. Clément, O. Stezowski, C. A. Ur, W. Urban, P. H. Regan, Z. Podolyák, C. Larijani, C. Townsley, R. Carroll, E. Wilson, H. Mach, L. M. Fraile, V. Paziy, B. Olaizola, V. Vedia, A. M. Bruce, O. J. Roberts, J. F. Smith, M. Scheck, T. Kröll, A.-L. Hartig, A. Ignatov, S. Ilieva, S. Lalkovski, N. Mărginean, T. Otsuka, N. Shimizu, T. Togashi, and Y. Tsunoda, Phys. Rev. C 96, 054323 (2017).

[34] J.-M. Régis, J. Jolie, N. Saed-Samii, N. Warr, M. Pfeiffer, A. Blanc, M. Jentschel, U. Köster, P. Mutti, T. Soldner, G. S. Simpson, F. Drouet, A. Vancraeyenest, G. de France, E. Clément, O. Stezowski, C. A. Ur, W. Urban, P. H. Regan, Z. Podolyák, C. Larijani, C. Townsley, R. Carroll, E. Wilson, L. M. Fraile, H. Mach, V. Paziy, B. Olaizola, V. Vedia, A. M. Bruce, O. J. Roberts, J. F. Smith, M. Scheck, T. Kröll, A.-L. Hartig, A. Ignatov, S. Ilieva, S. Lalkovski, W. Korten, N. Mărginean, T. Otsuka, N. Shimizu, T. Togashi, and Y. Tsunoda, Phys. Rev. C 95, 054319 (2017).
[35] Y. Khazov, A. Rodionov, and G. Shulyak, Nucl. Data Sheets 136, 163 (2016).

[36] J.-M. Régis, M. Dannhoff, and J. Jolie, Nucl. Instrum. Methods Phys. Res., Sect. A 897, 38 (2018).

[37] T. Kibédi, T. Burrows, M. Trzhaskovskaya, P. Davidson, and C. Nestor, Nucl. Instrum. Methods Phys. Res., Sect. A 589, 202 (2008).

[38] R. Orlandi, A. G. Smith, D. Patel, G. S. Simpson, R. M. Wall, J. F. Smith, O. J. Onakanmi, I. Ahmad, J. P. Greene, M. P. Carpenter, T. Lauritsen, C. J. Lister, R. V. F. Janssens, F. G. Kondev, D. Seweryniak, B. J. P. Gall, O. Dorveaux, and A. E. Stuchbery, Phys. Rev. C 73, 054310 (2006).

[39] Online ENDSF nuclear structure database, retrieved April 2018, http://www.nndc.bnl.gov/ensdf/.

[40] G. Lhersonneau and S. Brant, Phys. Rev. C 72, 034308 (2005).

[41] B. Pritychenko, M. Birch, B. Singh, and M. Horoi, At. Data Nucl. Data Tables 107, 1 (2016).

[42] J. M. Daugas et al. (unpublished).

[43] F. Iachello and O. Scholten, Phys. Rev. Lett. 43, 679 (1979).

[44] F. Iachello and P. van Isacker, The Interacting Boson-Fermion Model, Cambridge Monographs on Mathematical Physics (Cambridge University Press, Cambridge, 1991).

[45] F. Iachello and A. Arima, Phys. Lett. B 53, 309 (1974).

[46] A. Arima and F. Iachello, Phys. Rev. Lett. 35, 1069 (1975).

[47] J. E. Garcia-Ramos, K. Heyde, R. Fossion, V. Hellemans, and S. De Baerdemacker, Eur. Phys. J. A 26, 221 (2005).

[48] G. Lhersonneau, B. Pfeiffer, K.-L. Kratz, H. Ohm, K. Sistemich, S. Brant, and V. Paar, Z. Phys. A 337, 149 (1990).

[49] E. T. Gregor, M. Scheck, R. Chapman, L. P. Gaffney, J. Keatings, K. R. Mashtakov, D. O’Donnell, J. F. Smith, P. Spagnoletti, M. Thürauf, V. Werner, and C. Wiseman, Eur. Phys. J. A 53, 50 (2017). 